\title{
Social Stress Engages Opioid Regulation of Locus Coeruleus Norepinephrine Neurons and Induces a State of Cellular and Physical Opiate Dependence
}

\author{
Nayla N Chaijale*,', Andre L Curtis', Susan K Wood', Xiao-Yan Zhang', Seema Bhatnagar', \\ Beverly AS Reyes ${ }^{2}$, Elisabeth J Van Bockstaele ${ }^{2}$ and Rita J Valentino' \\ 'Division of Stress Neurobiology, Department of Anesthesiology, Abramson Research Center, The Children's Hospital of Philadelphia, Philadelphia, \\ PA, USA; '2Department of Neurosurgery, Thomas Jefferson University, Philadelphia, PA, USA
}

\begin{abstract}
Stress is implicated in diverse psychiatric disorders including substance abuse. The locus coeruleus-norepinephrine (LC-NE) system is a major stress response system that is also a point of intersection between stress neuromediators and endogenous opioids and so may be a site at which stress can influence drug-taking behaviors. As social stress is a common stressor for humans, this study characterized the enduring impact of repeated social stress on LC neuronal activity. Rats were exposed to five daily consecutive sessions of social stress using the resident-intruder model or control manipulation. LC discharge rate recorded 2 days after the last manipulation was decreased in stressed rats compared with controls. By 10 days after the last manipulation, LC rates were comparable between groups. Systemic administration of the opiate antagonist, naloxone, robustly increased LC discharge rate in a manner suggestive of opiate withdrawal, selectively in stressed rats when administered 2 or 10 days after the last manipulation. This was accompanied by behavioral signs of mild opiate withdrawal. Western blot and electron microscopic studies indicated that repeated social stress decreased corticotropin-releasing factor type I receptor and increased $\mu$-opioid receptor levels in the LC. Together, the results suggest that repeated social stress engages endogenous opioid modulation of LC activity and induces signs of cellular and physical opiate dependence that endure after the stress. These cellular effects may predispose individuals with a history of repeated social stress to substance abuse behaviors.

Neuropsychopharmacology (2013) 38, 1833-1843; doi:I0.1038/npp.2013.117; published online 19 June 2013
\end{abstract}

Keywords: resident-intruder; naloxone; opiate withdrawal; corticotropin-releasing factor; $\mu$-opioid receptor

\section{INTRODUCTION}

In response to perceived life-threatening stimuli, endocrine, physiological, and behavioral responses are initiated in a coordinated manner to cope with the challenge (McEwen, 1998). This 'stress response' is adaptive and important for survival. However, repeated or chronic engagement of stress response systems can have pathological consequences and predispose to psychiatric disorders such as depression, anxiety, post-traumatic stress disorder, and substance abuse (Berenz and Coffey, 2012; Gold and Chrousos, 2002; Heim and Nemeroff, 2001; McEwen, 1998). The locus coeruleus (LC)-norepinephrine (NE) system is a stress response system that has been implicated in heightened arousal and cognitive aspects of the stress response (Valentino and Van Bockstaele, 2008). Excessive activation of the LC-NE system has been linked to hyperarousal symptoms such as sleep

*Correspondence: Dr NN Chaijale, Division of Stress Neurobiology, Department of Anesthesiology, Abramson Research Center, Children's Hospital of Philadelphia, 3416 Civic Center Boulevard Suite 402, Philadelphia, PA 19104, USA, Tel: + 215590 0654, Fax: + I 215590 3364, E-mail: chaijalen@email.chop.edu

Received 5 November 2012; revised 8 May 2013; accepted 8 May 2013; accepted article preview online 10 May 2013 disturbances, inability to concentrate, ruminations, and/or increased vigilance that are shared by stress-related psychiatric diseases (Southwick et al, 1999; Wong et al, 2000).

In response to acute stress, LC activity is co-regulated by the stress-related neuropeptide, corticotropin-releasing factor (CRF), and the endogenous opioid, enkephalin (for review, see Valentino and Van Bockstaele, 2008). CRF axon terminals synapse with LC dendrites and CRF increases LC neuronal discharge rate (Curtis et al, 1997; Jedema and Grace, 2004; Van Bockstaele et al, 1996). Enkephalincontaining axon terminals also synapse with LC dendrites (Van Bockstaele et al, 1995) and act on $\mu$-opioid receptors (MORs) that are highly expressed by LC neurons to potently inhibit activity (Mansour et al, 1994; Williams and North, 1984). Notably, although LC neurons are also innervated by the $\kappa$-opioid receptor agonist, dynorphin, $\kappa$-opioid receptors are predominantly localized in axon terminals (Kreibich et al, 2008). Consistent with this presynaptic localization, $\kappa$-opiate receptor agonists do not alter LC spontaneous activity (Kreibich et al, 2008). Recordings of LC neuronal activity during acute stress indicate that stressors release both CRF and enkephalin to co-regulate LC activity in opposing manners. The net overall effect is a 
CRF-mediated excitation. However, removal of this influence with a CRF antagonist reveals a naloxone-mediated inhibition. This inhibition restrains the CRF activation and also facilitates a return toward baseline activity when the stressor is terminated (Curtis et al, 2001; Curtis et al, 2012).

In contrast to the well-described effects of acute stress on LC neuronal activity, there are few investigations of the effects of repeated or chronic stressors that more closely relate to psychopathology, on LC activity. One study demonstrated that repeated auditory stress desensitizes LC neurons to CRF and hypotensive stress (Conti and Foote, 1996), consistent with reported agonist- and stress-induced CRF type 1 receptor (CRF1) internalization (Reyes et al, 2006, 2008). A common stressor encountered by humans that has been implicated in psychiatric disease is social stress (Faravelli et al, 2012; Taylor et al, 2011). This has been modeled in rodents using the resident-intruder model, an ethologically relevant animal model of psychosocial stress that involves subjecting a male rat (intruder) to aggressive threats by a larger, unfamiliar male rat (resident) by placing it in the resident's home cage (Miczek, 1979). Although there are several modifications of this model, many studies demonstrate that repeated exposure to this stress produces endpoints of depression and anxiety in animal models and promotes drug self administration (Miczek et al, 2004; Rygula et al, 2005, Wood et al, 2010).

This study characterized the enduring effect of repeated social stress on rat LC activity using the resident-intruder model. LC neuronal discharge was recorded in the unanesthetized state before stress exposure and then 2 and 10 days after the final exposure. As social stress resulted in a decrease in LC rate, the role of endogenous opioids in this effect was further investigated.

\section{MATERIALS AND METHODS}

\section{Animals}

Adult male Sprague-Dawley rats were used as intruders (275-300 g) and male Long-Evans retired breeders were used as residents $(650-850 \mathrm{~g})$. All rats were ordered from Charles River (Wilmington, MA) and were individually housed in a controlled environment $\left(20^{\circ} \mathrm{C}, 12\right.$-h light-dark cycle, lights on at 0700 hours). Food and water were available ad libitum. Experiments were performed in the light cycle at approximately 1000 hours. Care and use of animals was approved by the Children's Hospital of Philadelphia Institutional Animal Care and Use Committee and in accordance with NIH Guide for the Care and Use of Laboratory Animals.

\section{Surgery}

Rats were anesthetized in $2 \%$ isoflurane-air mixture, positioned in a stereotaxic instrument, and surgically prepared for localization of the LC and subsequent implantation of an electrode array consisting of eight Teflon insulated $50 \mu \mathrm{m}$ stainless-steel microwires (NB Labs, Denison, TX), as previously described (Curtis et al, 2012; Kreibich et al, 2008). The array was attached to a Microstar head stage that was connected to a 16-channel data acquisition system (AlphaLab; Alpha Omega; Nazareth Illit,
Israel). After detecting LC activity, the multiwire array was affixed to the skull and screws with dental cement. The scalp wound was sutured closed. Animals were allowed 3 days of recovery before the experimental manipulations.

\section{Repeated Social Stress}

After post-surgical recovery, rats were randomly assigned to either a social stress or control group. A baseline of LC neuronal activity was recorded on the first day (day 1) before any manipulation. Rats assigned for repeated social stress were exposed to the resident-intruder stress as in our previous study (Wood et al, 2010). Intruder rats were placed into the cage of the resident. A period of investigation followed by an aggressive encounter by the resident usually ensued. When the intruder either assumed a supine posture signaling subordination for $3 \mathrm{~s}$ (defeat) or $15 \mathrm{~min}$ had elapsed from the time when the intruder was placed into the cage, the intruder and resident were separated by a wire mesh partition to prevent further physical contact but allow for exposure to auditory and olfactory stimuli for the remainder of a 30 -min session. Control rats were placed in a novel cage behind the partition for 30-min daily. All rats were returned to their home cage after each session. This was repeated daily for 5 consecutive days with the intruder being exposed to different residents each day. As previous studies in our laboratory demonstrated that rats cluster into two sub-populations based on their latency to assume defeat (Wood et al, 2010), the latency to defeat was noted for each subject.

\section{Electrophysiological Recordings}

All electrophysiological recordings were performed in the unanesthetized state in the home cage, in which rats had free movement. On the first experimental day, the implanted multiwire array was connected by cables to the data acquisition system and LC activity was recorded after a 10-min habituation period. Immediately after this baseline recording, the cable was disconnected and rats were exposed to 5 days of social stress or control manipulation. As the goal was to characterize the enduring effects of social stress on LC activity, neuronal recordings were obtained 2 days (day 7) or 10 days (day 15) after the last manipulation. To investigate the influence of endogenous opioids on LC activity, some rats were administered the opiate antagonist, naloxone $(2 \mathrm{mg} / \mathrm{kg}$, s.c.) 2 or 10 days after the last stress or control manipulation and LC activity was recorded before and after naloxone administration. In some experiments 2 days after the last manipulation, the selective CRF1 antagonist, NBI 30775 (10 mg/kg, s.c.) was administered $30 \mathrm{~min}$ before naloxone.

\section{Electrophysiological Analysis}

LC multiunit activity was recorded as continuous analog waveforms using the AlphaLab interfaced to a host computer (Alpha Omega, Alpharetta, GA), as previously described (Curtis et al, 2012; Kreibich et al, 2008). Multiunit activity on all eight wires (channels) was monitored in realtime and spike sorting of multiunit activity was done offline. The WaveMark template-matching algorithm in the 
Spike2 software (Cambridge Electronic Design, CED, v7.09) was used to discriminate putative LC single-unit waveforms. A set of waveforms identified by the WaveMark template is verified as events from a single unit by the principal component analysis and associated autocorrelograms (Figures 1a and b). Briefly, for principal component analysis, Spike2 generates a cluster of dots representing waveform events from a putative single unit in threedimensional space. An ellipsoid representing 1.5 SD from the cluster centrality is generated with the cluster of waveform events. The lack of overlap of any two ellipsoids was considered verification that the clusters were events from separate single units. An autocorrelogram of a set of waveform events in which no spikes occurred during an absolute refractory period of $2 \mathrm{~ms}$ or less was considered verification that those events were from a single unit. For each channel with LC activity, 2-5 single units were usually discriminated. LC activity was typically isolated on 2-4 channels in an individual rat. LC discharge rate from all units from all rats were averaged to obtain overall mean discharge rate from control and social stressed groups. In addition, to analyze the time course of the mean LC discharge rates, the discharge rates from all cells from each treatment group were averaged and plotted in 5-s bins.

\section{Histology}

At the end of the experiment, current was passed through the electrode $(10 \mu \mathrm{A}, 20 \mathrm{~s})$ and rats were perfused with $6 \%$ potassium ferrocyanide (in phosphate-buffered saline) to form a Prussian blue reaction product for identification of the electrode site. After perfusion, brains were removed and stored in $-80^{\circ} \mathrm{C}$. Frozen coronal sections $(30 \mu \mathrm{m})$ were cut through the LC and thaw-mounted onto slides, stained with Neutral red and the recording site was identified by microscopic visualization of the Prussian blue reaction product (Figure 1c). Data from rats with electrode localizations outside of the LC were not used in the analysis of neuronal activity.

\section{Behavioral Analysis}

For rats that were administered naloxone, behavior was recorded before and after naloxone injection and quantified by a treatment-blind observer as the incidence of specific behaviors including: chewing/teeth chattering, body/head shakes, grooming, rearing, and burying/digging. Behavioral observations were carried out in each rat's home cage.

\section{Western Blot}

Protein levels of CRF1 and MOR in LC tissue were quantified by western blotting as previously described (Bangasser et al, 2010). Rats were killed by decapitation $24 \mathrm{~h}$ after the last manipulation and extracted brains were snapfrozen in isopentane and placed at $-80{ }^{\circ} \mathrm{C}$ until used. Brains were blocked and sectioned in a cryostat to the level of caudal LC (identified as $-10 \mathrm{~mm}$ from Bregma using Paxinos and Watson Brain Atlas). The LC was microdissected bilaterally using a trephine $(1 \mathrm{~mm})$. These were pooled and stored at $-80^{\circ} \mathrm{C}$ until use. Tissue samples were homogenized, centrifuged, and supernatant was collected as a Templates given by the respective (color coded) cell clusters
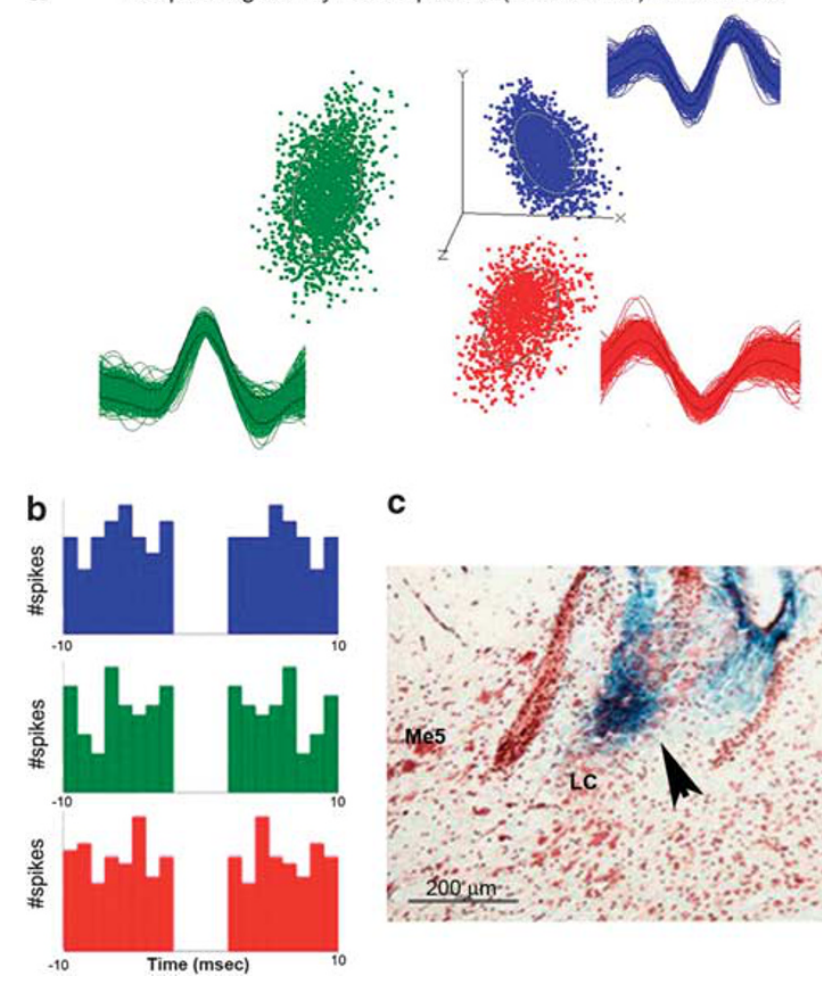

C

Figure I Discrimination of LC units and histology. (a, b) An example of three waveforms identified by the Wave-Mark template is verified as events from a single unit by the principal component analysis (a) and associated autocorrelograms (b). The lack of overlap of the ellipsoids (clusters) (a) and the lack of spikes occurred during an absolute refractory period of $2 \mathrm{~ms}$ or less (b) verified that these waveforms represent three different units. (c) Histological verification of a recording site in LC. The photomicrograph shows a coronal section through the LC. Slides were stained with Neutral red and the recording site was identified by microscopic visualization of the Prussian blue reaction product within the LC nucleus (arrow). Scale bar (bottom left) represents $200 \mu \mathrm{m}$. LC, locus coeruleus nucleus; Me5, mesencephalic trigeminal nucleus.

previously described (Bangasser et al, 2010). Protein content was determined using the BCA method. Protein extracts $(10 \mu \mathrm{g})$ were subjected to SDS-PAGE gel electrophoresis and transferred to polyvinylidene fluoride membranes (Immobilon-FL). Membranes were blocked with Odyssey Buffer (diluted in PBS 1:1) and incubated with the following primary antibodies: goat anti-CRF1/2 (1:500; Santa Cruz Biotech, Santa Cruz, CA), rabbit anti-MOR (1:1000; Invitrogen, Camarillo, CA), rabbit anti GAPDH (1:5000, Sigma-Aldrich, St Louis, MO), or mouse anti- $\beta$ actin, (1:5000, Sigma-Aldrich). After rinses, membranes were incubated with the following fluorescent secondary antibodies: donkey anti-goat for CRF1/2 (1:5000, IRDye $800 \mathrm{CW}$, LiCor), donkey anti-rabbit for detection of MOR (1:5000, IRDye $800 \mathrm{CW}$, LiCor), donkey-anti rabbit for GAPDH (1:10000, IRDye 680, LiCor), and donkey antimouse for $\beta$-actin ( $1: 10000$, IRDye 680 , LiCor). For loading controls, a fluorescent secondary antibody with a different infrared wavelength than the protein of interest was used for simultaneous detection of both primary antibodies. Membranes were scanned and Odyssey Infrared Imaging software quantified the integrated intensity of each band 
and determined molecular weights based on Bio-Rad Precision Plus Protein Standards. The CRF1:GAPDH and MOR: $\beta$-actin ratios were calculated and the mean ratios were compared between groups using ANOVAs. Duplicate or triplicate samples were averaged for an individual rat.

\section{Electron Microscopy}

Different groups of rats were used for electron microscopic visualization of CRF1 and MOR following previously described protocols (Reyes et al, 2006; Xu et al, 2004). Twenty-four hours after the last manipulation, rats were anesthetized with sodium pentobarbital $(60 \mathrm{mg} / \mathrm{kg})$ and perfused transcardially with $50 \mathrm{ml}$ of $3.8 \%$ acrolein (Electron Microscopy Sciences, Fort Washington, PA) and $200 \mathrm{ml}$ of $2 \%$ paraformaldehyde in $0.1 \mathrm{M}$ phosphate buffer (PB), $\mathrm{pH}$ 7.4. Brains were cut into $1-3 \mathrm{~mm}$ coronal slices, postfixed and sections $(40 \mu \mathrm{m})$ were cut through the LC using a Vibratome. The sections were incubated in rabbit anti-CRF1 antiserum $(\mathrm{H}-215 ; 1: 200)$ or rabbit anti-MOR antiserum $(1: 2500)$ for $15-18 \mathrm{~h}$ at room temperature. The specificity of the CRF1 antibody has been previously characterized with blocking peptide and in CRF1 knockout mice (Bangasser et al, 2010; Reyes et al, 2006, 2008). Control sections were processed identically with the exception of the omission of the primary antisera. Sections were incubated in goat antirabbit IgG ultra-small conjugate (Electron Microscopy Sciences). Silver intensification of the gold particles was achieved using a silver enhancement kit (Amersham Biosciences). Sections were flat embedded in Epon 812 (Electron Microscopy Sciences) and thin sections (80$100 \mathrm{~nm}$ ) were cut from the outer surface of the tissue, collected on grids, and counterstained with uranyl acetate and Reynolds lead citrate. Sections were examined with an electron microscope (Morgagni, Fei, Hillsboro, OR), and digital images were captured using the AMT advantage HR/ HR-B CCD camera system (Advance Microscopy Techniques, Danvers, MA). Figures were assembled and adjusted for brightness and contrast in Adobe Photoshop.

For quantification, tissue sections from at least 3 rats per group with optimal preservation of ultrastructural morphology were used. The ratio of cytoplasmic to total silver grains in a dendrite was determined for each dendrite (at least 300 and 100 dendrites for CRF1 and MOR count, respectively) and the mean ratio was determined per rat. The average of the ratio for each rat in an experimental group was taken as the group mean and these were compared between groups by an ANOVA.

\section{Statistical Analysis}

A two-way ANOVA was used to analyze changes in mean LC discharge rates between groups. A repeated-measure twoway ANOVA was used to analyze the time course of LC activity and cellular and behavioral responses after naloxone injection. A one-way ANOVA was used to analyze western blot and electron microscopy results. For all data analyses, the Student-Newman-Keuls test was used for post hoc comparisons of means. An alpha level of $p<0.05$ was the maximum threshold for statistical significance (Sigma Plot version 11.0, Systat Software, Chicago, IL).

\section{Drugs}

Naloxone (Sigma-Aldrich) was dissolved in saline $(2 \mathrm{mg} / \mathrm{ml})$, prepared fresh on each test day and administered subcutaneously in $2 \mathrm{mg} / \mathrm{kg}$ dose. NBI 30775 (Neurocrine Bioscience, San Diego, CA) was dissolved in a $10 \%$ solution of $0.1 \mathrm{M}$ tartaric acid in sterile water and administered as a $10 \mathrm{mg} / \mathrm{kg}$ dose (s.c.). The dose and pre-treatment time was chosen based on previous studies (Caille et al, 1999; Funk et al, 2007).

\section{RESULTS \\ Repeated Social Stress Decreases LC Activity}

Previous studies using rats without intracranial implants demonstrated that exposure to resident-intruder stress results in two populations of intruder rats, defined by the latency to assume a subordinate posture ( $>300$ or $<300 \mathrm{~s}$; Wood et al, 2010). In the present experiment, rats used for LC recordings did not cluster into two populations. Rather the latency to assume a supine posture was biased toward the longer duration $(599 \pm 66 ; n=13)$.

Figure 2a shows the time course of the mean LC discharge rates for control and stressed rats ( $n=8$ rats per group), on day $1(n=65$ cells and $n=53$ cells for control rats and stressed rats, respectively) and day 7 (2 days after the final experimental manipulation; $n=41$ cells in both, control and stressed rats). Discharge rates were stable and comparable between groups on day 1 (Figures 2a and c). Mean overall LC rates recorded on day 1 , before the first experimental manipulation were not different between groups (Figure 2c). By 2 days after the last manipulation (day 7), LC neuronal activity was significantly decreased in stressed rats as compared with control rats (Figure 2a). Across the time course of the recording, a two-way repeatedmeasures ANOVA showed a group by time interaction $(\mathrm{F}(120,8940)=4.43 ; p<0.001)$. A two-way ANOVA comparing the mean overall rates between groups on different days demonstrated a group by day interaction $(F(1,197)=$ 11.51, $p<0.001$; Figure 2c).

Figure $2 b$ shows the time course of the mean LC discharge rates for a different group of control and stressed rats $(n=3$ rats per group) on day 1 ( $n=15$ cells and $n=23$ cells for control and stressed rats, respectively) and day $15(n=12$ cells and $n=21$ cells for control and stressed rats, respectively). Discharge rates were stable and comparable between groups on day 1 (Figures $2 \mathrm{~b}$ and d). Similarly, the overall mean LC rates recorded on day 1 , before the first experimental manipulation were comparable (Figure 2d). On day 15, LC discharge rates were also similar between groups. Analysis of the entire time course of the recording revealed that there was no effect between the groups $(\mathrm{F}(1,2880)=0.04 ; p=0.84$; Figure $2 \mathrm{~b}$ ). A comparison of the overall mean rates on days 1 and 15 indicated that there was no effect of group or day $(\mathrm{F}(1,67)=0.61 ; p=0.44$; Figure $2 \mathrm{~d})$.

\section{Naloxone Selectively Activates LC Neurons of Rats Exposed to Repeated Social Stress}

Naloxone, administered on day 7, selectively increased LC discharge rates of stressed rats $(n=21$ cells per 4 rats $)$ 

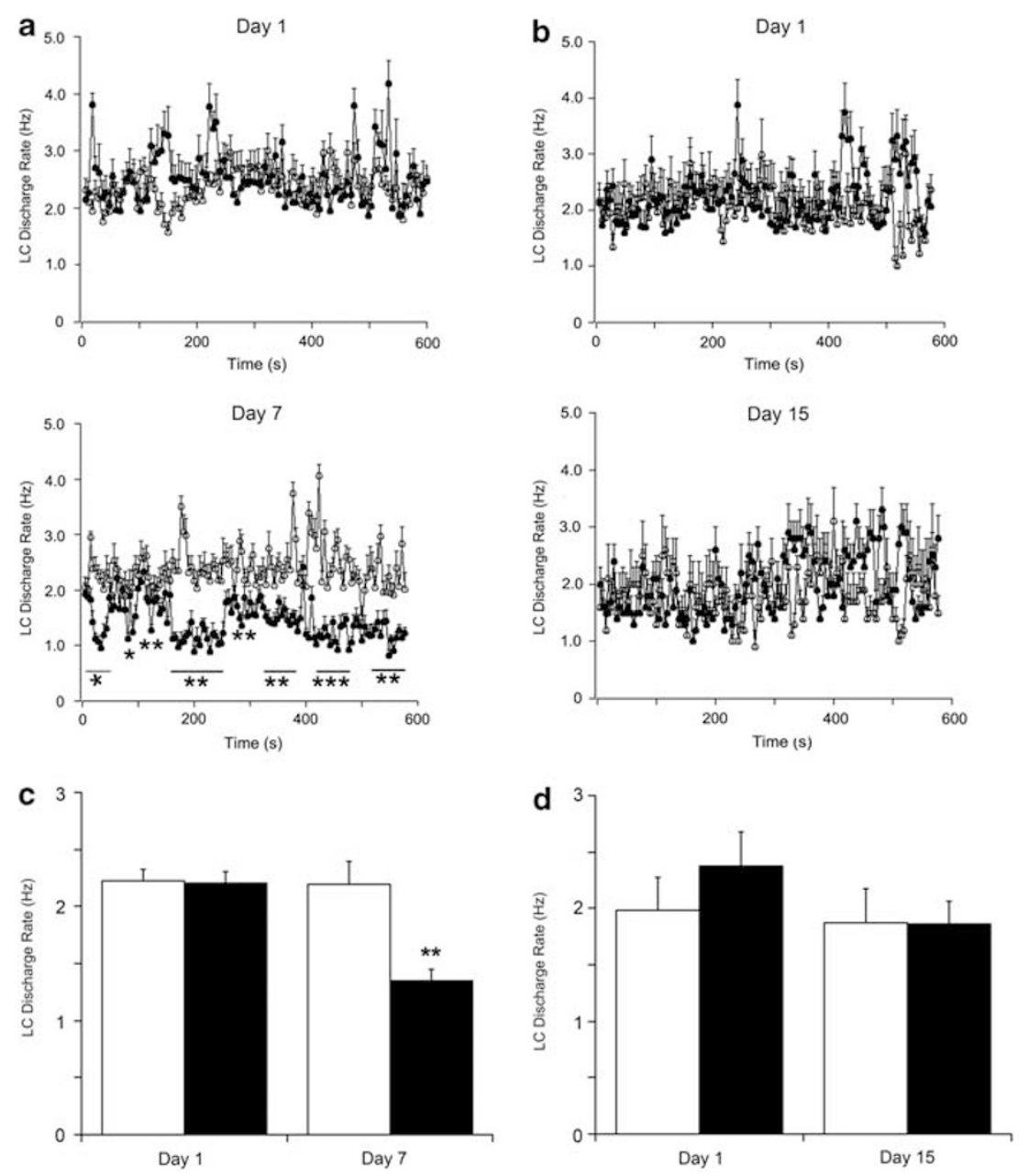

Figure 2 Effects of repeated social stress on LC discharge rate. (a) Time course of the mean LC discharge rates of control (open circles) and stressed rats (closed circles), determined over a 10-min recording on day I ( $n=65$ cells per 8 rats and $n=53$ cells per 8 rats for control and stressed rats, respectively) and day 7 ( $n=4 \mathrm{I}$ cells per 7 rats for control and stressed rats, respectively). The abscissae indicate time in second and the ordinates indicate mean LC discharge rate $(\mathrm{Hz})$. (b) Time course of the mean LC discharge rates of control (open circles) and stressed rats (closed circles), determined over a I0-min recording on day $\mid$ ( $n=15$ cells per 3 rats and $n=23$ cells per 3 rats for control and stressed rats, respectively) and day $I 5$ ( $n=12$ cells and $n=2 \mid$ cells, for control and stressed rats, respectively). The abscissae and the ordinates as indicated in a. (c) Bar graphs showing the mean LC discharge rates on day I and on day 7 for control (open bars) and stressed (closed bars) rats, respectively. (d) Bar graphs showing the mean LC discharge rates on day I and on day I 5 for control (open bars) and stressed (closed bars) rats, respectively. For all graphs, alpha values are represented as: $* * * *<0.001$, $* * p<0.01$, and $* p<0.05$. Note that the seven rats used for day I and day 7 (a) are different from those rats used on panel b (day I and day I5).

compared with control rats (17 cells per 4 rats; Figures 3a1 and b1). The increased LC neuronal activity occurred immediately after naloxone injection and was maintained above the level of control rats for at least $90 \mathrm{~s}$ (Figure 3a1). A two-way repeated-measures ANOVA over the time course revealed an effect of stress and naloxone injection $(\mathrm{F}(120,3236)=4.11 ; p<0.001)$. A comparison of the mean overall discharge rates before and during the $200 \mathrm{~s}$ after naloxone revealed an effect of stress and naloxone $(\mathrm{F}(1,36)=28.9, p<0.001)$. Before naloxone, the mean discharge rate was lower in stressed compared with control rats and this increased following naloxone administration. In contrast, the mean discharge rates before and after naloxone were not different for control rats (Figure $3 \mathrm{~b} 1$ ).

LC sensitivity to naloxone in rats exposed to repeated social stress endured at least 10 days after the last stress exposure (day 15, Figures $3 \mathrm{a} 2$ and $\mathrm{b} 2$ ). Figure $3 \mathrm{a} 2$ shows the time course of the LC discharge rate recorded in control (12 cells per 3 rats) and social stressed rats ( 21 cells per 3 rats) on day 15, before and after naloxone injection. Naloxone increased LC neuronal activity selectively in stressed rats and this increase was maintained for nearly $5 \mathrm{~min}$ (Figure 3a2). A two-way repeated-measures ANOVA over the time course indicated an effect of stress and naloxone injection $(\mathrm{F}(120,3751)=7.40 ; p<0.001)$. Similarly, a comparison of the mean rates before and after naloxone injection revealed an effect of stress and naloxone $(\mathrm{F}(1,31)=38.11, p<0.001$; Figure $3 \mathrm{~b} 2)$. Rates were comparable before naloxone and increased only for stressed rats after naloxone injection. Notably, the magnitude of the naloxone-induced increase was larger on day 15 compared with day 7 (Figures $3 a$ and $b$ ).

To determine whether the naloxone-elicited increase in LC activity in stressed rats was due to an unmasking of a 

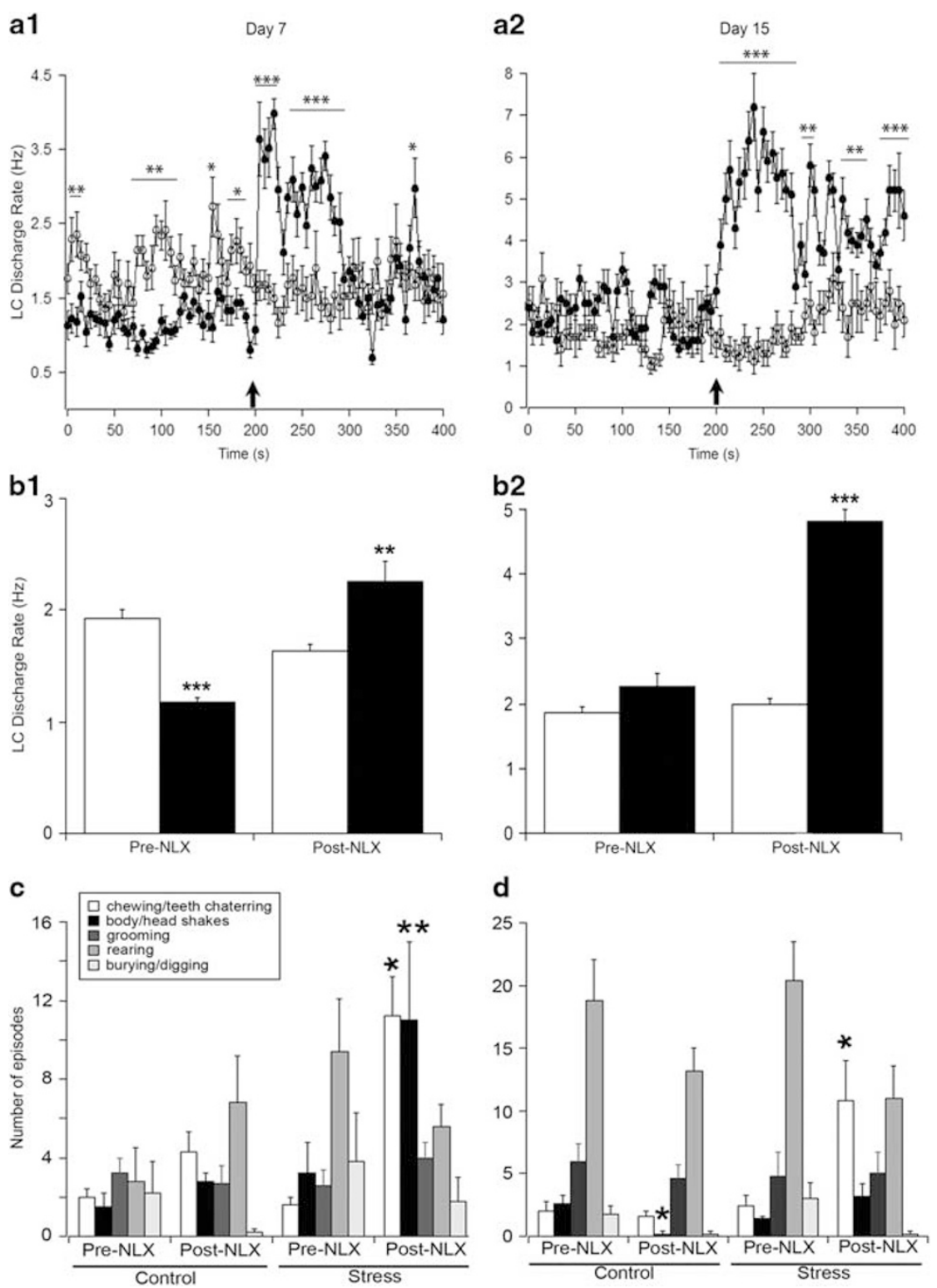

Figure 3 Effects of naloxone (NLX) on LC discharge rate and behavior. (al) Time course of the mean discharge rates of control (open circles, 17 cells per 4 rats) and stressed rats (closed circles, 21 cells per 4 rats) on day 7, before and after NLX. (a2) Time course of the mean discharge rates of control (open circles, 12 cells per 3 rats) and stressed rats (closed circles, 2 I cells per 3 rats) on day 15, before and after NLX. In both, day 7 and day I5, NLX is administered at $200 \mathrm{~s}$, as indicated by the arrow. (b l, b2) Bar graphs represent the mean LC discharge rate of control (white bars) and stress (black bars) rats, on day 7 and day 15, before and after NLX injection. (c) Mean incidence of opiate withdrawal-related behaviors observed before (pre-) and after (post-) NLX injection to control $(n=6)$ and stressed $(n=5)$ rats on day 7. (d) Mean incidence of opiate withdrawal-related behaviors observed before (pre-) and after (post-) NLX injection to control $(n=5)$ and stressed $(n=5)$ rats on day I5. For all graphs, asterisks represent $* * * *<<0.00$ I, $* * p<0.0$ I, and $* p<0.05$.

CRF tonic activation, the selective CRF1 antagonist, NBI 30775, was administered before naloxone administration in stressed rats on day 7. For these subjects, the mean baseline LC discharge rates on day 1 and day 7 were $2.0 \pm 0.2$ and $1.6 \pm 0.1 \mathrm{~Hz}$, respectively ( $n=12$ cells per 2 rats). Following NBI 30775 and before naloxone administration, LC rates were similar $1.4 \pm 0.1 \mathrm{~Hz}$. Naloxone still increased LC discharge rate in rats pre-treated with NBI 30775 to a mean of $3.4 \pm 0.3 \mathrm{~Hz} \quad(\mathrm{~F}(1,11)=6.8, p<0.05)$, similar to the effect of naloxone on day 7 in rats not pre-treated with NBI 30775 (see Supplementary Material and Supplementary Figure S1).

\section{Naloxone Elicits Opiate Withdrawal Signs Selectively in Rats Exposed to Repeated Social Stress}

Naloxone administration on day 7 elicited behaviors indicative of opiate withdrawal in stressed rats, including chewing/teeth chattering $(\mathrm{F}(1,7)=6.9, p<0.01)$ and body and headshakes $(\mathrm{F}(1,7)=19.3, p<0.01)$. The incidence of these and other scored behaviors before naloxone administration was not different between groups $(p=0.87$; Figure $3 c$ ). Similar signs were observed in rats that were pre-treated with NBI 30775. Although naloxone-elicited body and head shakes subsided in the stressed rats by 
day 15 , the number of episodes of chewing/teeth chattering behavior remained elevated in stressed compared with control rats $(\mathrm{F}(1,8)=7.6, p<0.05$; Figure $3 \mathrm{~d})$.

\section{Repeated Social Stress Alters CRF1 and MOR Levels and Trafficking in the LC}

Rats used for these studies were not implanted with electrodes. Examination of latency to defeat revealed both long and short latency populations $(533.11 \pm 49.8$ and $218.33 \pm 26.4$ for long and short latency rats, respectively).

Repeated social stress increased MOR levels in the LC $(\mathrm{F}(2,23)=5.1, p<0.02)$. This increase was selective to rats that were characterized by a relatively long latency to defeat (comparable to stressed rats with LC recordings; Figure 4a). In contrast to MOR, CRF1 protein levels decreased in the LC of rats exhibiting long latency to defeat $(\mathrm{F}(2,22)=2.87$, $p<0.05)$ and tended to be decreased for short latency rats $(p=0.09$; Figure $4 \mathrm{~b})$.

Figure 5a shows examples of immunogold labeled MOR in LC dendrites. Consistent with the results of the western blot experiments, quantification of the number of immunogold labeled MOR particles/dendrite revealed that this was greater in social stress rats that were characterized by a long latency to defeat ( $3.8 \pm 0.1, n=3$ rats) compared with control rats $(2.4 \pm 0.1, n=3$ rats) and short latency rats $(2.4 \pm 0.1, n=3, \mathrm{~F}(2,8)=52, p<0.0005)$. In addition, there was a greater ratio of cytoplasmic:total immunogold MOR labeling, indicative of MOR re-distribution (eg, internalization) in the social stress group characterized by long latency to defeat $(\mathrm{F}(2,8)=8.7, p<0.02$; Figure $5 \mathrm{a}$, bar graph). Interestingly, this was not apparent in social stressed rats that were characterized by a short latency to defeat (Figure 5a, bar graph).
Figure $5 \mathrm{~b}$ shows examples of CRF1 immunogold labeling in LC dendrites of control ( $n=8$ rats) and stressed rats. For rats exposed to social stress that were characterized by a long latency to defeat ( $n=7$ rats), there was no difference in the ratio of cytoplasmic:total CRF1 labeling. However, evidence for CRF1 internalization was apparent in social stress rats that were characterized by a short latency to defeat ( $n=7$ rats) as indicated by a greater cytoplasmic:total CRF1 ratio $(\mathrm{F}(2,21)=8.7, p<0.005)$.

\section{DISCUSSION}

This study demonstrated that repeated social stress produces enduring changes in LC neuronal activity. In contrast to LC excitation that is associated with acute stress, repeated social stress produced an inhibition that was apparent at $48 \mathrm{~h}$ after stress termination and returned to baseline by 10 days. The naloxone-elicited LC activation that occurred selectively in stressed rats implicated endogenous opioids in this effect. Consistent with this, MOR levels were increased, particularly in the cytoplasmic compartment of LC neurons, as would be predicted by enkephalin release. The engagement of endogenous opioid regulation of the LC resulted in a state of cellular opioid dependence as indicated by the findings that naloxone increased LC discharge to rates greater than those seen in control rats and that naloxone-elicited LC activation also occurred at a time when LC discharge was no longer inhibited in stressed rats. The naloxone-elicited LC activation is reminiscent of that produced in animals that have been chronically administered morphine (Aston-Jones et al, 1997; Rasmussen et al, 1990; Valentino and Wehby, 1989). Naloxone also elicited physical signs of opiate withdrawal selectively in social stressed rats. Together, the findings
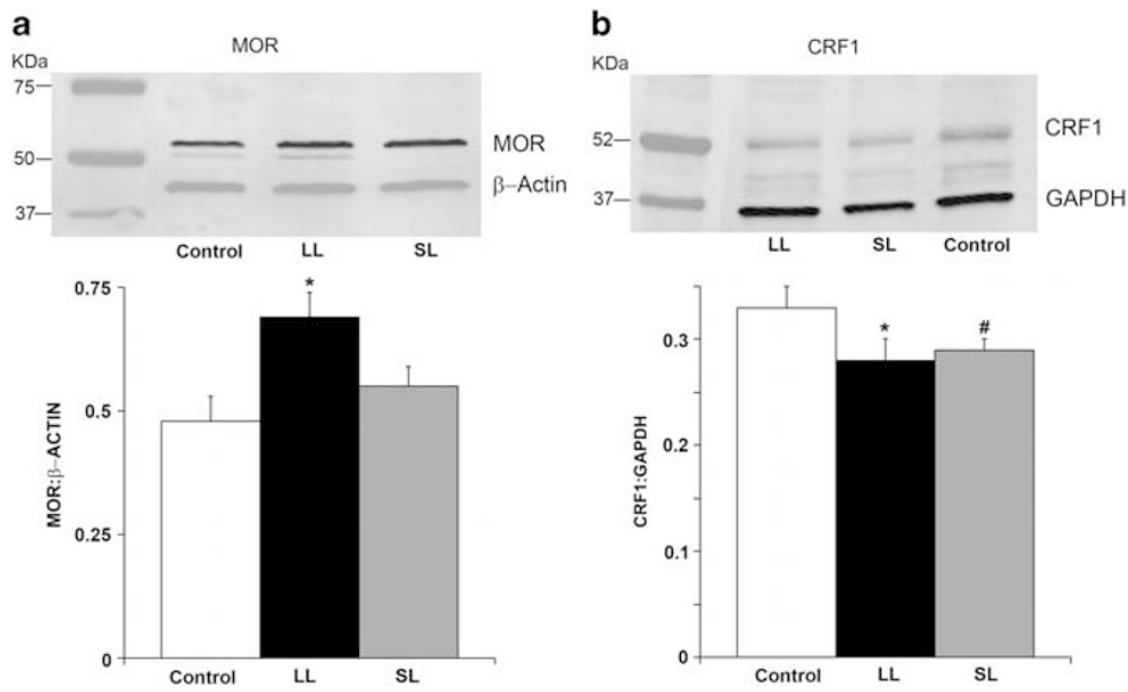

Figure 4 Western blot analysis of CRF receptor I (CRFI) and $\mu$-opioid receptor (MOR) in the locus coeruleus (LC), in control rats or rats that went through repeated social stress and were classified as short (SL) or long latency (LL). (a) Representative blot showing protein expression of MOR $(\mathrm{MW}=70 \mathrm{kDa})$ and $\beta$-actin $(\mathrm{MW}=42 \mathrm{kDa})$. Bar graphs show the mean ratio of the integrated intensity of each band of $\mathrm{MOR}$ protein to the corresponding control band from the same sample. There was a difference of MOR expression in LC from $\mathrm{LL}(n=6)$ rats compared with control $(n=7)$ rats $(F(2,2 I)=5.15, * p>0.0 I)$. SL $(n=I I)$ rats did not show any differences from control rats $(p=0.25)$. (b) Representative blot showing protein expression of CRFI receptor $(\mathrm{MW}=52 \mathrm{kDa})$ and $\mathrm{GAPDH}(\mathrm{MW}=36 \mathrm{kDa})$. Bar graphs show the mean ratio of the integrated intensity of each band of $\mathrm{CRF}$ I protein to the corresponding control band from the same sample. ANOVA analysis showed a decrease in CRFI receptor expression between LL $(n=6)$ rats and control $(n=8)$ rats $\left(F(2,22)=2.87,{ }^{*} p>0.05\right)$. SL $(n=1 I)$ rats tended to be decreased as compared with control rats $\left({ }^{\#} p=0.09\right)$. 
a

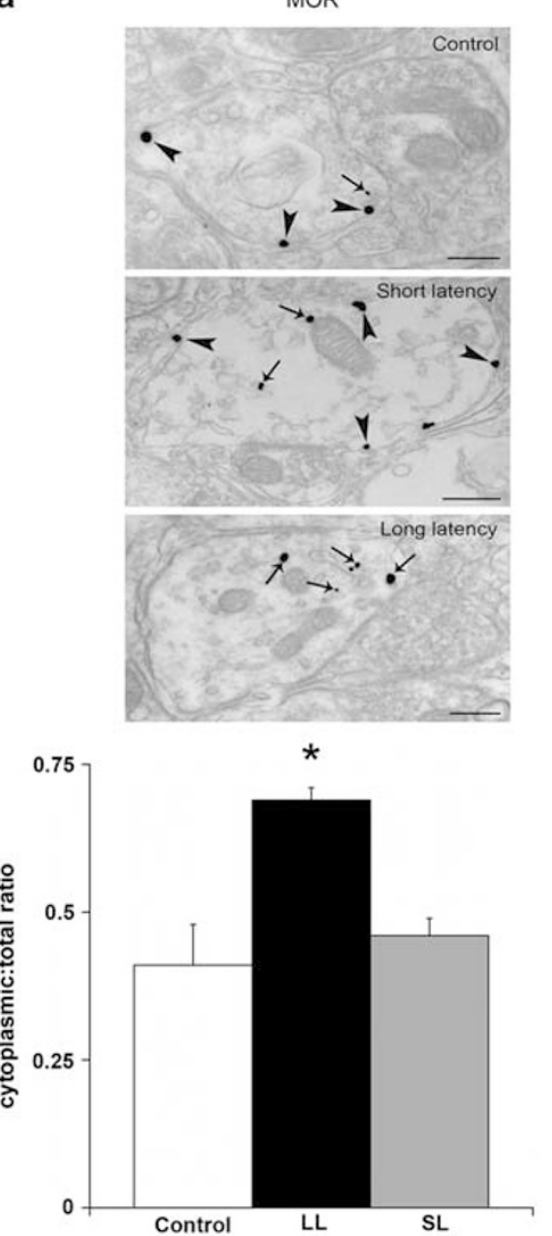

b
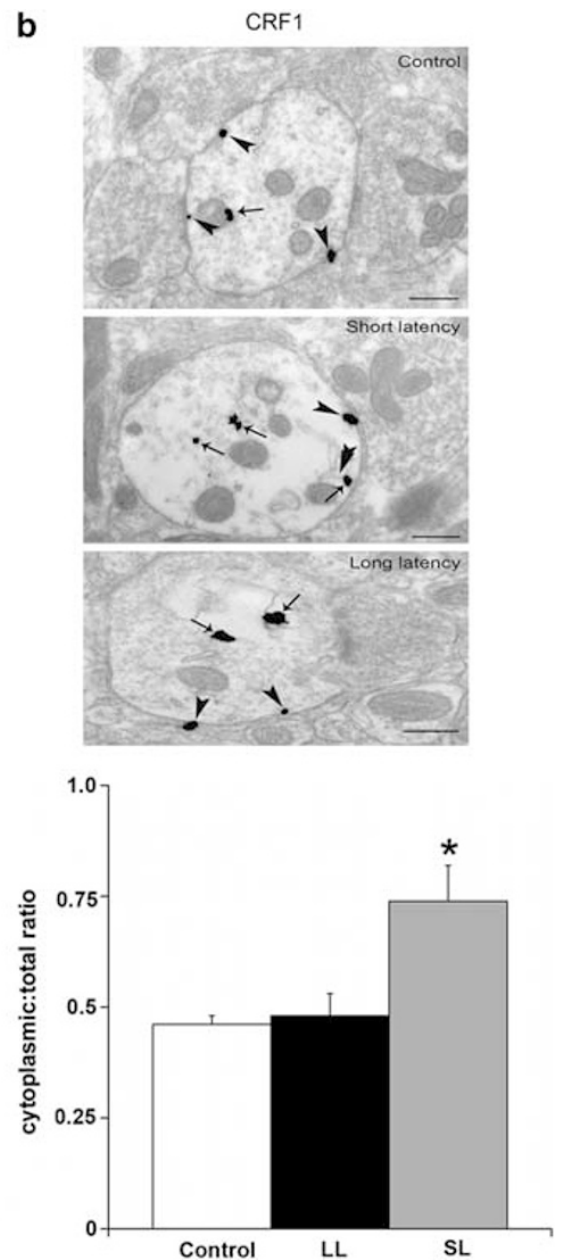

Figure 5 Electron microscopic evidence for repeated social stress-induced re-distribution of (a) $\mu$-opioid receptor (MOR) and (b) CRF receptor I (CRFI) in rat LC. Panel a-top shows a dendrite from a control rat that contains immunogold-silver labeling for MOR (arrowheads) along the plasmalemma. MOR within the cytoplasmic compartment is depicted by an arrow. Panel a-center demonstrates MOR labeling in a dendrite from a rat exhibiting short latency behavior (SL). Immunogold-silver labeling for MOR (arrowheads) can be seen primarily along the plasmalemma, although some MOR-immunogoldsilver particles are localized within the cytoplasmic compartment. Panel a-bottom illustrates that immunogold-silver labeling for MOR shifts from the plasmalemma to the cytoplasm in long latency rats (LL). Immunogold-silver labeling for MOR is shown within the cytoplasmic compartment as depicted by arrows, whereas arrowheads point to immunogold-silver labeling on the plasma membrane. Bar graph from panel a shows quantitative representation of the ratio of cytoplasmic to total immunogold-silver labeling of MOR in LC for control rats, SL rats and $L L$ rats $(n=3$ rats per group, $>100$ dendrites per rat analyzed). ANOVA analysis for MOR internalization showed a difference between groups $(F(2,6)=8.7 \mathrm{I}$; $* 2<0.05)$, indicating that there was an increased percent internalization of MOR only in LL rats. Panel b-top shows a dendrite from a control rat that contains immunogold-silver labeling for CRF I (arrowheads) along the plasmalemma. An arrow depicts CRFI within the cytoplasmic compartment. Panel b-center illustrates that immunogold-silver labeling for CRFI shifts from the plasmalemma to the cytoplasm in rats that exhibit short-latency behavior following exposure to repeated social stress. Immunogold-silver labeling for CRFI is shown within the cytoplasmic compartment as depicted by arrows. A CRFI is also found along the plasmalemma (arrowhead). A double headed arrowhead indicates an endosome-like vesicle associated with CRFI. Panel b-bottom shows a dendrite from a rat exhibiting long-latency behavior following exposure to repeated social stress. CRFI distribution in LC from rats that exhibited long latency behavior was similar to CRFI distribution in LC from control rats. Quantitative representation of the ratio of cytoplasmic:total CRFI immunogold label (panel b, bar graph) indicated that CRFI was internalized in $\mathrm{SL}(n=7)$ rats $(F(2,19)=8.812 ; * p<0.01)$, but not in $L L(n=7)$ or control $(n=8)$ rats $(>300$ dendrites per rat analyzed). Scale bars: $0.5 \mu \mathrm{m}$.

suggest that repeated social stress induces an enduring opioid regulation of the brain LC-NE system and a state of cellular and physical opiate dependence. These neural changes may confer vulnerability to opiate abuse in subjects with a history of social stress.

\section{Acute Stress and LC Activity}

Acute stressors of different modalities activate the LC-NE system as indicated by recordings of LC neuronal activity, c-fos expression in LC neurons, and NE release in LCforebrain targets (Chan and Sawchenko, 1994; Curtis et al, 2012; Smagin et al, 1994). Evidence has implicated CRF as the neurotransmitter that mediates stress-elicited LC-NE activation. CRF axon terminals synapse with LC dendrites and intra-LC CRF administration mimics the effects of acute stressors on LC discharge (Curtis et al, 1997; Van Bockstaele et al, 1996). Administration of CRF antagonists directly into the LC prevents stress-induced activation of the LC-NE system and unmasks a naloxone-sensitive inhibition that is 
apparent either during stress or immediately following stress termination (Curtis et al, 2001; Valentino et al, 1991). When both opiate and CRF receptors are blocked, LC neurons are non-responsive to acute stress, suggesting that these are the major afferents that regulate the LC-NE system during stress (Curtis et al, 2012). Together, these findings suggest a model whereby the LC is regulated in opposing manners by CRF and endogenous MOR ligands during acute stress (Valentino and Van Bockstaele, 2008). In this model, stress engages CRF afferents to the LC to bias activity to a high tonic state that promotes increased arousal and behavioral flexibility. At the same time, endogenous opioids are released to exert an inhibitory influence that restrains the excitation and facilitates recovery on stressor termination.

\section{Repeated Social Stress and LC Activity}

In contrast to LC activation by acute stressors, repeated social stress resulted in lower LC discharge rates compared with the control manipulation when measured $48 \mathrm{~h}$ after the last stress. A similar inhibition has been described with repeated auditory stress or repeated CRF when LC activity was recorded $24 \mathrm{~h}$ after the last stress (Conti and Foote, 1995, 1996). Given evidence that acute stressors engage an opioid inhibitory influence on the LC, this was a likely candidate for the inhibition seen after repeated social stress. The robust naloxone-elicited LC activation of rats exposed to repeated social stress confirmed a role for endogenous opioids in the relatively long-lasting inhibition of LC activity. The activation was not due to an unmasking of a CRF excitation because it occurred in rats that were pretreated with a CRF antagonist. In addition, CRF receptors were downregulated in stressed rats. Naloxone-elicited LC activation likely represents a cellular withdrawal response, similar to that seen when morphine-dependent rats are administered an opiate antagonist (Aston-Jones et al, 1997; Rasmussen et al, 1990; Valentino and Wehby, 1989). The finding that naloxone still elicits a robust LC activation by 10 days after repeated stress, at a time when baseline LC neuronal discharge rates are no longer inhibited, indicates that repeated stress produces a state of cellular tolerance and dependence. The observation of naloxone-elicited physical signs resembling opiate withdrawal at both 2 and 10 days after the final stressor was consistent with this. These results are reminiscent of studies demonstrating that rats and mice exposed to repeated resident-intruder stress develop an analgesia that is cross-tolerant with morphine and that lasts for months (Miczek, 1991; Miczek et al, 1986). Similarly, in mice resident-intruder stress produced a state of opiate physical dependence as evidenced by naloxoneprecipitated withdrawal jumping that was of a similar magnitude as that produced in morphine-dependent mice (Miczek et al, 1986). Together with the present findings, these studies underscore the ability of social stress to bring endogenous opioid systems online to a sufficient extent as to produce physical dependence.

\section{LC Hyperactivity and Behavioral Withdrawal}

In morphine-dependent rats, opiate antagonist administration directly onto LC neurons by iontophoresis or microinfusion produces withdrawal hyperactivity (Aghajanian, 1978; Aston-Jones et al, 1997). Whether this effect is due to intrinsic mechanisms within LC neurons or to excitatory afferents has remained controversial (Akaoka and AstonJones, 1991; Rasmussen and Aghajanian, 1989). Currently, it is accepted that both intrinsic and extrinsic mechanisms contribute to the opiate antagonist-precipitated withdrawal hyperactivity of LC neurons of morphine-dependent animals. In this study using the microwire array, it was not feasible to directly inject naloxone into the LC so it is not known for certain whether naloxone-elicited LC activation of the stressed rats was the result of direct activation of LC neurons or activation of LC afferents. Similarly, the link between LC activation and withdrawal behaviors is not definitively established. Previous lesion studies suggested that the LC is not necessary for withdrawal behaviors (Caille et al, 1999; Delfs et al, 2000). However, other studies involving direct inhibition of LC activity or manipulation of cyclic AMP signaling in the LC provide evidence for its involvement in opiate withdrawal signs (Han et al, 2006; Mirzaii-Dizgah et al, 2008; Punch et al, 1997; Taylor et al, 1998). Even if not necessary, LC activation is sufficient to elicit opiate withdrawal signs as indicated by the findings that the antagonist is most potent at eliciting physical withdrawal signs when administered directly into the LC (Maldonado et al, 1992). Nonetheless, the finding that naloxone-precipitated LC activation is still very strong at 10 days after the last stressor but the withdrawal signs were weaker suggests the involvement of other circuits, at least with respect to head and body shakes.

\section{Social Stress Effects on MOR and CRF1 in the LC}

As rats from which LC recordings were obtained were only characterized by long latencies to exhibit defeat, the relationship between stress effects on neuronal activity and receptors can only be interpreted for this subpopulation. Nonetheless, certain comparisons of stressrelated receptor changes between long and short latency rats lead to interesting speculation. In general, the findings suggest that repeated social stress results in a decrease in CRF influence on the LC as a result of receptor downregulation. The finding that $\mathrm{CRF} 1$ was downregulated in long latency rats without evidence of internalization may indicate that at this time CRF1 internalization and downregulation had already occurred and, although fewer in number, the receptors were redistributed equally between the cytoplasm and plasma membrane. The observation that the short latency rats exhibited CRF1 internalization and only a tendency for CRF1 downregulation suggest that they lag behind the long latency rats in the processes of CRF1 internalization and downregulation. Importantly, the downregulation of CRF1 could contribute to a shift from CRF excitatory influence to an opioid inhibitory regulation of the $\mathrm{LC}$ with repeated stress. The increase in MOR levels in LC detected by both western blot and electron microscopy and apparent MOR internalization in rats exposed to social stress that were characterized by a long latency to defeat would be consistent with an increased enkephalinergic drive to the LC in these rats. This could account for enhanced inhibitory tone of LC neurons and development of cellular dependence. That this is not seen in the short latency rats at 
this time could also be due to a lag in the processes regulating MOR in response to social stress. Alternatively, these processes may be impaired in this sub-population and this may have a role in the different phenotype. These questions lie beyond the scope of the article but inspire much future research.

\section{Clinical Implications}

We previously suggested that disturbing the opioid-CRF balance that regulates the LC during stress has potential clinically relevant consequences (Valentino and Van Bockstaele, 2008). For example, chronic morphine administration sensitized LC neurons to CRF and stress and this was expressed as a hyperactive coping style (Xu et al, 2004). This study implied that chronic opiates could increase vulnerability to post-traumatic stress disorder, which is characterized by hyperarousal. Conversely, this study suggests that repeated social stress could predispose to opiate abuse by creating a state of cellular dependence where exogenous opiates would serve as negative reinforcers to alleviate withdrawal hyperactivity of the LC system. Although the social stress model used in this study has been demonstrated to promote self-administration of cocaine and other stimulants, effects on opiate self-administration have not been as well studied. One study indicated that social stress history did not influence heroin self-administration (Cruz et al, 2011). However, social stress effectively reinstates morphine conditioned place preference in rats to a similar extent as physical stress and cues related to morphine withdrawal share this effect (Ribeiro Do Couto et al, 2006). Notably in humans, a history of child abuse is associated with opiate abuse (Schiff et al, 2010). The present findings suggest that inducing a state of cellular dependence within the LC-NE system may be one mechanism by which repeated social stress increases vulnerability to opiate abuse.

\section{FUNDING AND DISCLOSURE}

All authors declare no conflict of interest in relation to the work described. Drs Valentino, Van Bockstaele and Bhatnagar were compensated by the NIH for consulting services. This work was supported by DARPA 58077 LSDRP, DA09082, T32 NS007413 and a NARSAD Young Investigator Award (SKW). The CRF antagonist NBI 30775 was obtained from Neurocrine Bioscience, San Diego, CA.

\section{ACKNOWLEDGEMENTS}

The authors acknowledge the technical assistance of Elizabeth Verhoeve and Sandra Luz.

\section{REFERENCES}

Aghajanian GK (1978). Tolerance of locus coeruleus neurones to morphine and suppression of withdrawal response by clonidine. Nature 276: 186-188.

Akaoka H, Aston-Jones G (1991). Opiate withdrawal-induced hyperactivity of locus coeruleus neurons is substantially mediated by augmented excitatory amino acid input. J Neurosci 11: $3830-3839$.
Aston-Jones G, Hirata H, Akaoka H (1997). Local opiate withdrawal in locus coeruleus in vivo. Brain research 765: 331-336.

Bangasser DA, Curtis A, Reyes BA, Bethea TT, Parastatidis I, Ischiropoulos $\mathrm{H}$ et al (2010). Sex differences in corticotropinreleasing factor receptor signaling and trafficking: potential role in female vulnerability to stress-related psychopathology. Mol Psychiatry 15: 896-904.

Berenz EC, Coffey SF (2012). Treatment of co-occurring posttraumatic stress disorder and substance use disorders. Curr Psychiatry Rep 14: 469-477.

Caille S, Espejo EF, Reneric JP, Cador M, Koob GF, Stinus L (1999). Total neurochemical lesion of noradrenergic neurons of the locus ceruleus does not alter either naloxone-precipitated or spontaneous opiate withdrawal nor does it influence ability of clonidine to reverse opiate withdrawal. J Pharmacol Exp Ther 290: 881-892.

Chan RKW, Sawchenko PE (1994). Spatially and temporally differentiated patterns of c-fos expression in brainstem catecholaminergic cell groups induced by cardiovascular challenges in the rat. J Comp Neurol 348: 433-460.

Conti LH, Foote SL (1995). Effects of pretreatment with corticotropin-releasing factor on the electrophysiological responsivity of the locus coeruleus to subsequent corticotropin-releasing factor challenge. Neuroscience 69: 209-219.

Conti LH, Foote SL (1996). Reciprocal cross-desensitization of locus coeruleus electrophysiological responsivity to corticotropin-releasing factor and stress. Brain Res 722: 19-29.

Cruz FC, Quadros IM, Hogenelst K, Planeta CS, Miczek KA (2011). Social defeat stress in rats: escalation of cocaine and 'speedball' binge self-administration, but not heroin. Psychopharmacology 215: $165-175$.

Curtis AL, Bello NT, Valentino RJ (2001). Endogenous opioids in the locus coeruleus function to limit the noradrenergic response to stress. J Neurosci 21: RC152.

Curtis AL, Florin-Lechner SM, Pavcovich LA, Valentino RJ (1997). Activation of the locus coeruleus noradrenergic system by intracoerulear microinfusion of corticotropin-releasing factor: effects on discharge rate, cortical norepinephrine levels and cortical electroencephalographic activity. J Pharmacol Exp Ther 281: 163-172.

Curtis AL, Leiser SC, Snyder K, Valentino RJ (2012). Predator stress engages corticotropin-releasing factor and opioid systems to alter the operating mode of locus coeruleus norepinephrine neurons. Neuropharmacology 62: 1737-1745.

Delfs JM, Zhu Y, Druhan JP, Aston-Jones G (2000). Noradrenaline in the ventral forebrain is critical for opiate withdrawal-induced aversion. Nature 403: 430-434.

Faravelli C, Lo Sauro C, Lelli L, Pietrini F, Lazzeretti L, Godini L et al (2012). The role of life events and HPA axis in anxiety disorders: a review. Curr Pharmaceutical Design 18: 5663-5674.

Funk CK, Zorrilla EP, Lee MJ, Rice KC, Koob GF (2007). Corticotropin-releasing factor 1 antagonists selectively reduce ethanol self-administration in ethanol-dependent rats. Biol Psychiatry 61: 78-86.

Gold PW, Chrousos GP (2002). Organization of the stress system and its dysregulation in melancholic and atypical depression: high vs low CRH/NE states. Mol Psych 7: 254-275.

Han MH, Bolanos CA, Green TA, Olson VG, Neve RL, Liu RJ et al (2006). Role of cAMP response element-binding protein in the rat locus ceruleus: regulation of neuronal activity and opiate withdrawal behaviors. J Neurosci 26: 4624-4629.

Heim C, Nemeroff CB (2001). The role of childhood trauma in the neurobiology of mood and anxiety disorders: preclinical and clinical studies. Biol Psychiatry 49: 1023-1039.

Jedema HP, Grace AA (2004). Corticotropin-releasing hormone directly activates noradrenergic neurons of the locus ceruleus recorded in vitro. J Neurosci 24: 9703-9713. 
Kreibich A, Reyes BA, Curtis AL, Ecke L, Chavkin C, Van Bockstaele EJ et al (2008). Presynaptic inhibition of diverse afferents to the locus ceruleus by kappa-opiate receptors: a novel mechanism for regulating the central norepinephrine system. J Neurosci 28: 6516-6525.

Maldonado R, Stinus L, Gold LH, Koob GF (1992). Role of different brain structures in the expression of the physical morphine withdrawal syndrome. J Pharmacol Exp Ther 261: 669-677.

Mansour A, Fox CA, Thompson RC, Akil H, Watson SJ (1994). mu-Opioid receptor mRNA expression in the rat CNS: comparison to mu-receptor binding. Brain research 643: 245-265.

McEwen BS (1998). Stress, adaptation, and disease. Allostasis and allostatic load. Ann N Y Acad Sci 840: 33-44.

Miczek KA (1979). A new test for aggression in rats without aversive stimulation: differential effects of d-amphetamine and cocaine. Psychopharmacology (Berl) 60: 253-259.

Miczek KA (1991). Tolerance to the analgesic, but not discriminative stimulus effects of morphine after brief social defeat in rats. Psychopharmacology 104: 181-186.

Miczek KA, Covington HE 3rd, Nikulina EM Jr, Hammer RP (2004). Aggression and defeat: persistent effects on cocaine selfadministration and gene expression in peptidergic and aminergic mesocorticolimbic circuits. Neurosci Biobehav Rev 27: 787-802.

Miczek KA, Thompson ML, Shuster L (1986). Analgesia following defeat in an aggressive encounter: development of tolerance and changes in opioid receptors. Ann N Y Acad Sci 467: 14-29.

Mirzaii-Dizgah I, Karimian SM, Hajimashhadi Z, Riahi E, Ghasemi $\mathrm{T}$ (2008). Attenuation of morphine withdrawal signs by muscimol in the locus coeruleus of rats. Behav Pharmacol 19: 171-175.

Punch LJ, Self DW, Nestler EJ, Taylor JR (1997). Opposite modulation of opiate withdrawal behaviors on microinfusion of a protein kinase A inhibitor versus activator into the locus coeruleus or periaqueductal gray. J Neurosci 17: 8520-8527.

Rasmussen K, Aghajanian GK (1989). Withdrawal-induced activation of locus coeruleus neurons in opiate-dependent rats: attenuation by lesions of the nucleus paragigantocellularis. Brain Res 505: 346-350.

Rasmussen K, Beitner-Johnson DB, Krystal JG, Aghajanian GK, Nestler EJ (1990). Opiate withdrawal and rat locus coeruleus: behavioral, electophysiological and biochemical correlates. J Neurosci 10: 2308-2317.

Reyes BA, Fox K, Valentino RJ, Van Bockstaele EJ (2006). Agonistinduced internalization of corticotropin-releasing factor receptors in noradrenergic neurons of the rat locus coeruleus. Eur $J$ Neurosci 23: 2991-2998.

Reyes BA, Valentino RJ, Van Bockstaele EJ (2008). Stress-induced intracellular trafficking of corticotropin-releasing factor receptors in rat locus coeruleus neurons. Endocrinology 149: 122-130.

Ribeiro Do Couto B, Aguilar MA, Manzanedo C, Rodriguez-Arias M, Armario A, Minarro J (2006). Social stress is as effective as physical stress in reinstating morphine-induced place preference in mice. Psychopharmacology 185: 459-470.
Rygula R, Abumaria N, Flugge G, Fuchs E, Ruther E, HavemannReinecke U (2005). Anhedonia and motivational deficits in rats: impact of chronic social stress. Behav Brain Res 162: 127-134.

Schiff M, Levit S, Cohen-Moreno R (2010). Childhood sexual abuse, post-traumatic stress disorder, and use of heroin among female clients in Israeli methadone maintenance treatment programs (MMTPS). Social Work Health Care 49: 799-813.

Smagin GN, Swiergiel AH, Dunn AJ (1994). Sodium nitroprusside infusions activate cortical and hypothalamic noradrenergic systems in rats. Neurosci Res Comm 14: 85-91.

Southwick SM, Bremner JD, Rasmusson A, Morgan CA 3rd, Arnsten A, Charney DS (1999). Role of norepinephrine in the pathophysiology and treatment of posttraumatic stress disorder. Biol Psychiatry 46: 1192-1204.

Taylor JR, Punch LJ, Elsworth JD (1998). A comparison of the effects of clonidine and CNQX infusion into the locus coeruleus and the amygdala on naloxone-precipitated opiate withdrawal in the rat. Psychopharmacology (Berl) 138: 133-142.

Taylor PJ, Gooding P, Wood AM, Tarrier N (2011). The role of defeat and entrapment in depression, anxiety, and suicide. Psychological Bull 137: 391-420.

Valentino RJ, Page ME, Curtis AL (1991). Activation of noradrenergic locus coeruleus neurons by hemodynamic stress is due to local release of corticotropin-releasing factor. Brain Res 555: 25-34.

Valentino RJ, Van Bockstaele E (2008). Convergent regulation of locus coeruleus activity as an adaptive response to stress. Eur J Pharmacol 583: 194-203.

Valentino RJ, Wehby RG (1989). Locus coeruleus discharge characteristics of morphine-dependent rats: Effects of naltrexone. Brain Res 488: 126-134.

Van Bockstaele EJ, Branchereau P, Pickel VM (1995). Morphologically heterogeneous met-enkephalin terminals form synapses with tyrosine hydroxylase containing dendrites in the rat nucleus locus coeruleus: an immuno-electron microscopic analysis. J Comp Neurol 363: 423-438.

Van Bockstaele EJ, Colago EE, Valentino RJ (1996). Corticotropinreleasing factor-containing axon terminals synapse onto catecholamine dendrites and may presynaptically modulate other afferents in the rostral pole of the nucleus locus coeruleus in the rat brain. J Comp Neurol 364: 523-534.

Williams JT, North RA (1984). Opiate receptor interactions on single locus coeruleus neurons. Mol Pharm 26: 489-497.

Wong ML, Kling MA, Munson PJ, Listwak S, Licinio J, Prolo P et al (2000). Pronounced and sustained central hypernoradrenergic function in major depression with melancholic features: relation to hypercortisolism and corticotropin-releasing hormone. Proc Natl Acad Sci USA 97: 325-330.

Wood SK, Walker HE, Valentino RJ, Bhatnagar S (2010). Individual differences in reactivity to social stress predict susceptibility and resilience to a depressive phenotype: role of corticotropin-releasing factor. Endocrinology 151: 1795-1805.

Xu GP, Van Bockstaele E, Reyes B, Bethea T, Valentino RJ (2004). Chronic morphine sensitizes the brain norepinephrine system to corticotropin-releasing factor and stress. J Neurosci 24: 8193-8197.

Supplementary Information accompanies the paper on the Neuropsychopharmacology website (http://www.nature.com/npp) 